\title{
Glucagon-like peptide-1 receptor regulates endoplasmic reticulum stress-induced apoptosis and the associated inflammatory response in chondrocytes and the progression of osteoarthritis in rat
}

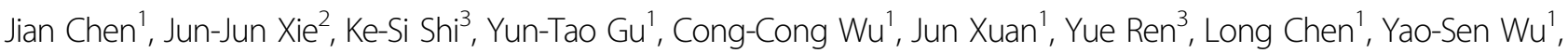 \\ Xiao-Lei Zhang ${ }^{1}$, Jian Xiao ${ }^{2}$, De-Zhong Wang ${ }^{4}$ and Xiang-Yang Wang ${ }^{1}$
}

\begin{abstract}
Treatments for osteoarthritis $(\mathrm{OA})$ are designed to restore chondrocyte function and inhibit cell apoptosis. Previous studies have shown that activation of the glucagon-like peptide-1 receptor (GLP-1R) leads to anti-inflammatory and anti-apoptotic effects. However, the role of GLP-1R in the pathological process of OA is unclear. In present work, we aimed to demonstrate the potential effect of GLP-1R on chondrocytes and elucidate its underlying mechanisms. We found that activation of GLP-1R with liraglutide could protect chondrocytes against endoplasmic reticulum stress and apoptosis induced by interleukin (IL)-1 $\beta$ or triglycerides (TGs). These effects were partially attenuated by GLP-1R small interfering RNA treatment. Moreover, inhibiting PI3K/Akt signaling abolished the protective effects of GLP-1R by increase the apoptosis activity and ER stress. Activating GLP-1R suppressed the nuclear factor kappa-B pathway, decreased the release of inflammatory mediators (IL-6, tumor necrosis factor a), and reduced matrix catabolism in TGtreated chondrocytes; these effects were abolished by GLP-1R knockdown. In the end, liraglutide attenuated rat cartilage degeneration in an OA model of knee joints in vivo. Our results indicate that GLP-1R is a therapeutic target for the treatment of $\mathrm{OA}$, and that liraglutide could be a therapeutic candidate for this clinical application.
\end{abstract}

\section{Introduction}

Osteoarthritis (OA) is a prevalent progressive and degenerative joint disease that results in personal activity limitations, disability, and great economic burden worldwide $^{1}$. Various factors contribute to the initiation and

\footnotetext{
Correspondence: D-Z. Wang (dzwang0624@yahoo.com) or X-Y. Wang (xiangyangwang@wmu.edu.cn)

${ }^{1}$ Department of Orthopaedics, The Second Affiliated Hospital and Yuying Children's Hospital of Wenzhou Medical University, Wenzhou 325027, People's Republic of China

${ }^{2}$ School of Pharmaceutical Sciences, Key Laboratory of Biotechnology and Pharmaceutical Engineering, Wenzhou Medical University, Wenzhou 325027 Zhejiang, China

Full list of author information is available at the end of the article

Jian Chen and Jun-Jun Xie contributed equally to this work.

Edited by B. Zhivotovsky
}

progression of OA, such as age, gender, body weight, heredity, and mechanical injury ${ }^{2,3}$. The main treatment for OA has been to delay its development; few antiinflammatory therapies have been used to relieve the symptoms of OA. Therefore, fully understanding the pathogenesis of OA would be helpful in exploring novel therapies.

Several studies have demonstrated inflammation-related changes in $\mathrm{OA}$ cartilage. The expression of proinflammatory mediators, including interleukin-1 (IL-1), IL-6, and tumor necrosis factor $\alpha$ (TNF- $\alpha$ ), increase in cartilage, bone, and synovium, contributing to the initiation of $\mathrm{OA}^{4,5}$. These cytokines activate the nuclear factor me. The images or other third party material in this article are included in the article's Creative Commons license, unless indicated otherwise in a credit line to the material. material is not included in the article's Creative Commons license and your intended use is not permitted by statutory regulation or exceeds the permitted use, you will need to obtain permission directly from the copyright holder. To view a copy of this license, visit http://creativecommons.org/licenses/by/4.0/. 


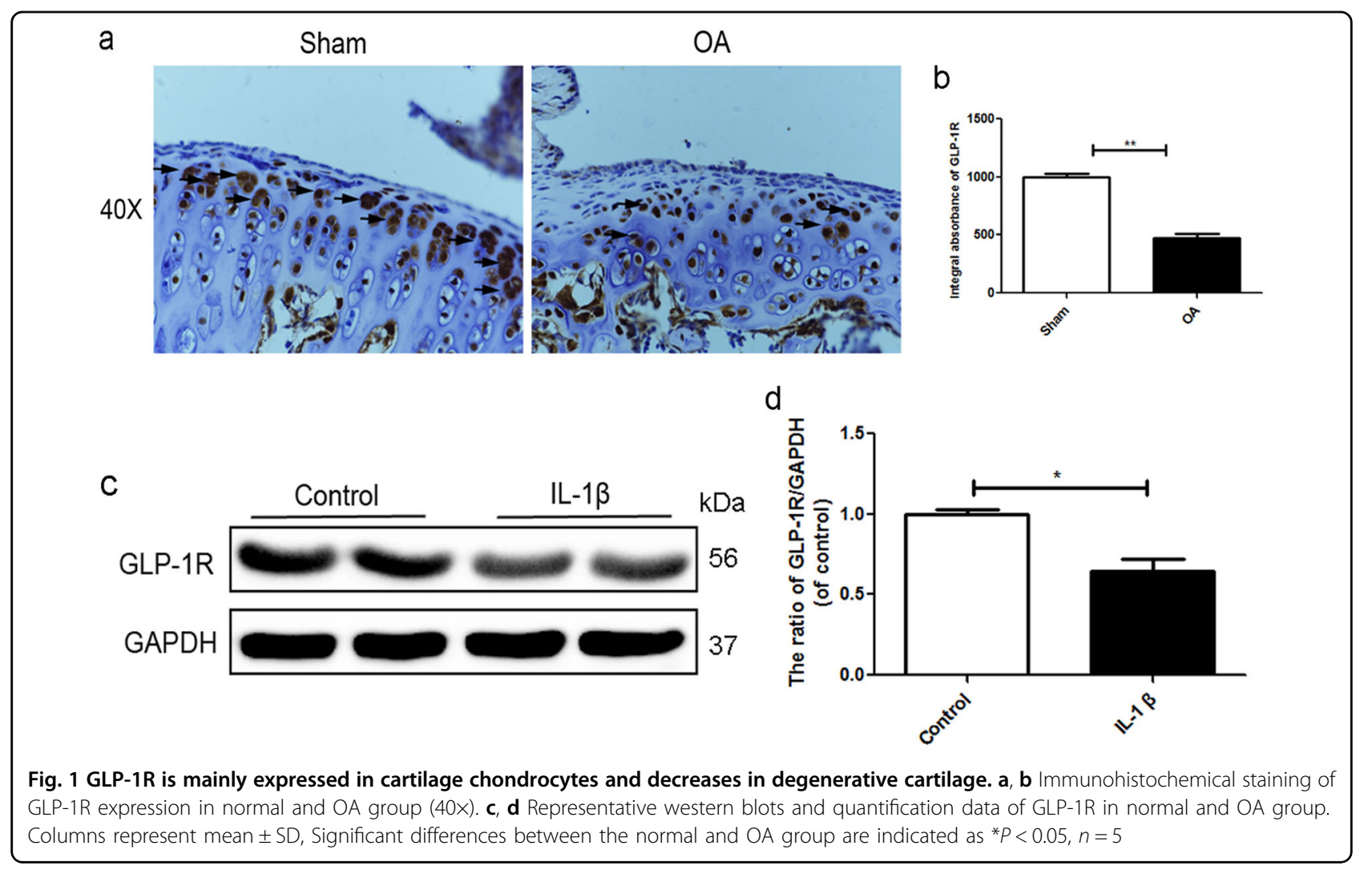

kappa-B (NF-kB) signaling pathway,inducing the upregulation of apoptotic proteins and matrix-degrading enzymes, such as metalloproteinases (MMPs), which irreversibly degrade the extracellular matrix $(\mathrm{ECM})^{6,7}$. A previous study showed that systematic blockade of IL-6 reduced expression of MMPs and ADAMTSs (a disintegrin and metalloproteinase with thrombospondin motifs) could alleviate medial meniscus-induced OA cartilage lesions ${ }^{8}$.

Endoplasmic reticulum (ER) stress is another critical etiology in the OA pathological process ${ }^{9-11}$. Accumulation of misfolded proteins in the ER triggers the unfolded protein response (UPR), in which C/EBP homologous protein $(\mathrm{CHOP})$ is activated, subsequently activating the caspase family and inducing chondrocyte death ${ }^{12}$. ATF6, a regulator of ER stress, can augment XBP1S gene expression and modulate ER stress-mediated apoptosis via caspase-3 and caspase-12 in OA cartilage ${ }^{13}$. In addition, it has been reported that the pro-inflammatory factor IL- $1 \beta$ induces ER stress-related apoptosis in cultured chondrocytes ${ }^{14}$. Furthermore, IL- $1 \beta$ plays a critical role in the initiation of $\mathrm{OA}$; evidence suggests that IL-1 $\beta$ can accelerate anabolic activities in chondrocytes ${ }^{15}$. In this study, IL-1 $\beta$ was used to induce ER stress and subsequent apoptosis.

Glucagon-like peptide-1 (GLP-1) is an incretin hormone, produced by enteroendocrine cells ( $\mathrm{L}$ cells) to regulate glucose and energy homeostasis via GLP-1R binding ${ }^{16}$. Not only does this have functional implications for diabetes mellitus, but it also has potential protective effects in the cardiovascular and nervous systems. GLP-1 can decrease cardiomyocyte destruction and increase cell viability after ischemia-reperfusion injury ${ }^{17}$. Exendin-4, a GLP-1R agonist, significantly promoted locomotor recovery in rats after spinal cord injury via inducing autophagy and inhibiting apoptosis ${ }^{18}$. Importantly, recent studies showed that activation of GLP-1R inhibited ER stress in several models. GLP-1R agonizts prevented the onset of ER stress and subsequent apoptosis in human umbilical vein endothelial cells when stimulated by high glucose $^{19}$. Additionally, a GLP-1R antagonist enhanced ER stress-related apoptosis in high glucose-stimulated HK-2 cells ${ }^{20}$.

However, to our knowledge, the role of GLP-1R in OA has not been reported to date. We hypothesized that the GLP-1R agonist, liraglutide, might alleviate chondrocyte apoptosis and ECM degradation by regulating ER stress. Moreover, activation of GLP-1R may be involved in ER stress-induced inflammatory responses. We also analysed the protective effect of liraglutide on cartilage degeneration in vivo. These new insights suggest that GLP-1R may be a potential target for the development of novel drugs in the treatment of OA. 


\section{Results}

GLP-1R is mainly expressed in cartilage chondrocytes and decreases in degenerative cartilage

Few articles have reported the role of GLP-1R in the articular cartilage degeneration. As shown in Fig. 1a, b, immunohistochemical staining and corresponding quantification of GLP-1R showed that GLP-1R of membrane is slightly but significantly downregulated in degenerative cartilage compared with normal cartilage. We used IL1- $\beta$ to mimic $\mathrm{OA}$ in chondrocytes in vitro. Western blotting data demonstrated that the expression of GLP-1R was lower in response to IL-1 $\beta$ stimulation than control group (Fig. 1c, d). Together, these results suggested a potential role of GLP-1R in OA.

\section{Activation of GLP-1R by liraglutide decreases apoptosis in chondrocytes}

To assess the role of GLP-1R in chondrocytes, we used liraglutide, a GLP-1R agonist, to activate GLP-1R with various concentrations. As shown in Fig. 2a, liraglutide exerted no significant cytotoxic effect on chondrocytes. And we noted that liraglutide concentrations up to $10^{-7}$ $M$ showed significant protective effect on IL-1 $\beta$ treatedchondrocytes (Fig. 2b). Chondrocytes shrunk in size, and reduced in number when exposed to IL-1 $\beta$, which was partially reversed by liraglutide (Fig. 2c). The western blotting analysis also showed that activation of GLP-1R significantly decreased the level of pro-apoptotic protein cleaved-caspase 3 and Bax and increased the level of antiapoptotic protein Bcl-2, compared with IL-1 $\beta$ inducedchondrocytes (Fig. 2d-g). Take together, these results showed that GLP-1R may exert an anti-apoptotic effect on chondrocytes.

\section{The anti-apoptotic effects of GLP-1R was modulated by PI3K/Akt signaling}

GLP-1 analogs regulate various biological processes by activating GLP-1R/PI3K/Akt signaling ${ }^{21,22}$. Interestingly, Western blotting demonstrated that GLP-1R activation further upregulated the ratio of $\mathrm{p}$-Akt/t-Akt when compared to IL-1 $\beta$ group (Fig. 3a, b). To explore whether the protective effects of GLP-1R were modulated by PI3K/Akt signal pathway, LY294002, a specific PI3K inhibitor was used to treat chondrocytes combined with liraglutide treatment. TUNEL staining showed that IL-1 $\beta$ markedly enhanced the apoptotic level, when compared to the control group. And GLP-1R activation significantly decreased apoptotic activity, which were reversed partially by LY294002 (Fig. 3c, d). Furthermore, Western blot assays noted increasing expression of apoptotic proteins active caspase 3 and Bax in the IL-1 $\beta$ group, which was abolished by liraglutide. Conversely, liraglutide upregulated the expression of anti-apoptotic marker Bcl-2 compare to IL-1 $\beta$ group. Furthemore, LY294002 abolished GLP-1R's anti-apoptotic effect (Fig. 3c-e). To further demonstrate the relationship between GLP-1R and PI3K/Akt signaling, GLP-1R siRNA was used to treat chondrocytes. And GLP-1R knockdown markedly abolished the effect of liraglutde to modulate the ratio of $\mathrm{p}$ Akt/t-Akt after liraglutide treatment, when compared with both the control and negative control siRNA group (Supplementary Figure S1a and c). Together, these results demonstrated that PI3K/Akt signaling was involved in the protective effect of GLP-1R.

\section{Activation of GLP-1R inhibits ER stress and associated apoptosis via activation of PI3K/Akt signaling in chondrocytes}

To test whether ER stress was related to the antiapoptotic effect of GLP-1R, the GRP78, PDI, caspase12 and $\mathrm{CHOP}$, which regarded as the markers of ER stress, were assessed by western blotting. As shown in Fig. 4a-e, IL-1 $\beta$ significantly increased the ER stress related protein. However, activation of GLP-1R by liraglutide attenuated the IL- $1 \beta$ induced-upregulation of ER stress-related proteins in chondrocytes. These results indicated that the protective role of GLP-1R may involve the inhibition of ER stress-related protein.

To further clarify the specific mechanism of GLP-1R in chondrocytes, we used the LY294002 to treat chondrocytes with liraglutide. Similarly, inhibition of PI3K/Akt signaling abolished the protective effect by liraglutide, indicating that PI3K/Akt signaling was involved in the GLP-1R' effects of inhibition of ER stress (Fig. 4f-j). To further demonstrate whether GLP-1R was associated with ER stress in cartilage, we used TG, a classic ER stress inducer, to cause ER stress and GLP-1R siRNA was used to knockdown the GLP-1R in chondrocytes. And western bolt results noted that activation of GLP-1R inhibited the TG-induced the increase of the expression of GRP78, PDI, CHOP and active caspase12 compared to control group, which were partially reversed by GLP-1R siRNA (Supplementary Figure S2a-e). In order to further demonstrate that ER stress is involved in the anti-apoptotic effect of GLP-1R, TUNEL assay was performed to assess the level of apoptotic cells. Compared with the TG treatment group, activation of GLP-1R by liraglutide markedly decreased the number of apoptotic cells. However, GLP1R knockdown greatly reduced anti-apoptotic activity of liraglutide (Supplementary Figure S3a and b). Moreover, the western blot results showed treatment of activating GLP-1R greatly inhibited the increase of Bax and cleaved caspase 3 and the decrease of Bcl-2 induced by TG. However, GLP-1R siRNA weakened the effect of liraglutide (Supplementary Figure S3c-f). These results further proved that GLP-1R activation contributes to the inhibition of ER stress and subsequent apoptosis resulted from GLP-1R activation. 
a

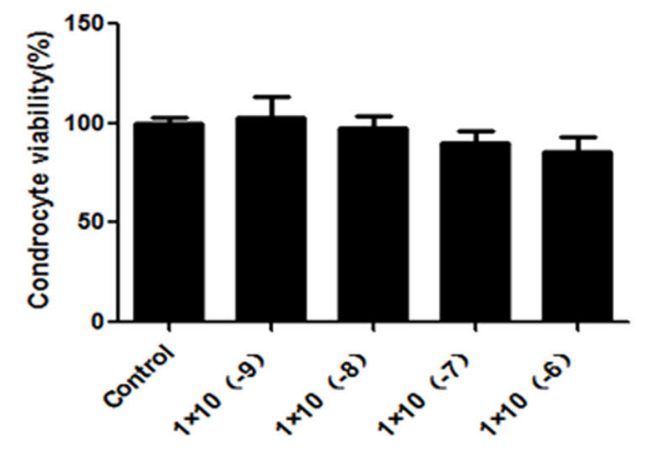

b

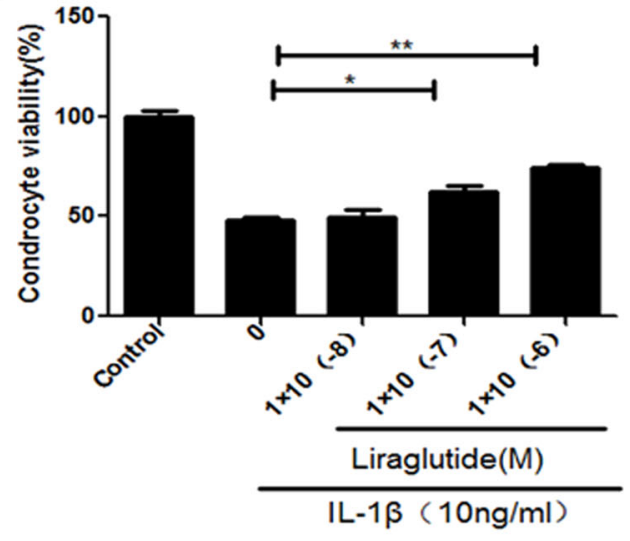

C

$\mathrm{IL}-1 \beta(10 \mathrm{ng} / \mathrm{ml})$
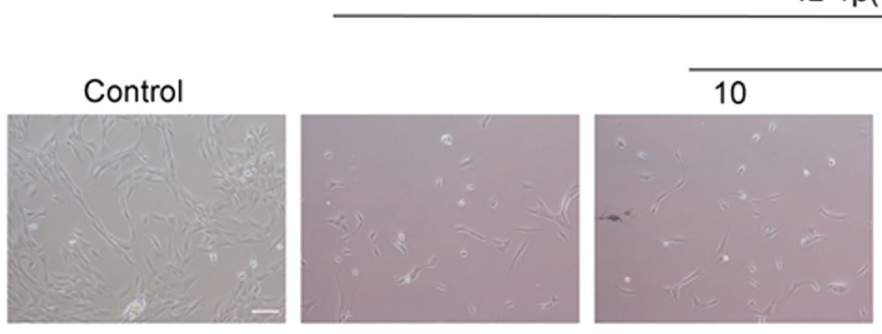

d

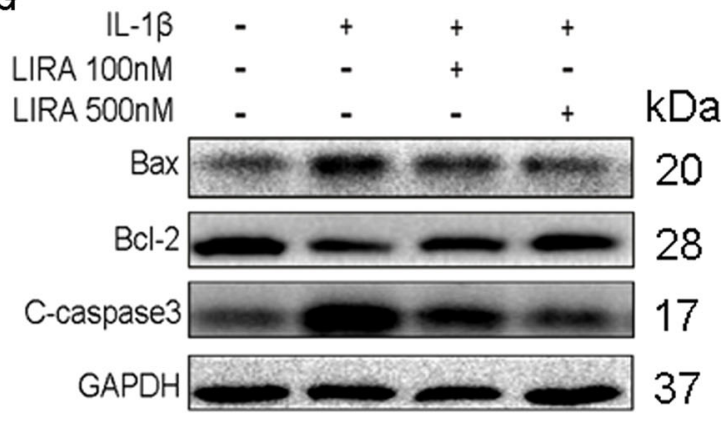

e

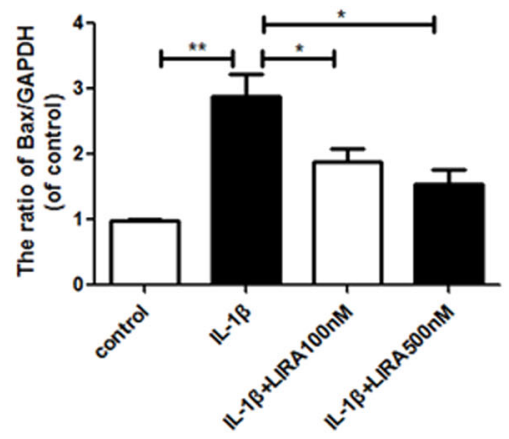

f

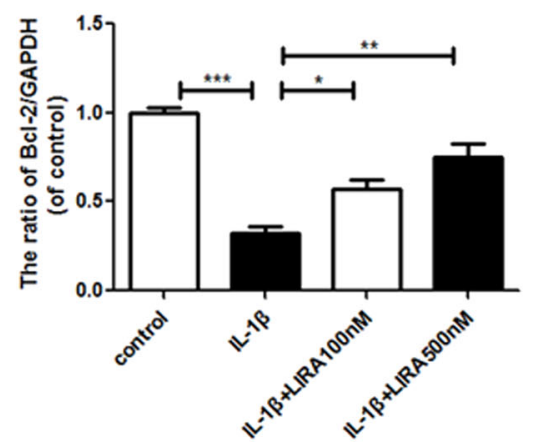

g

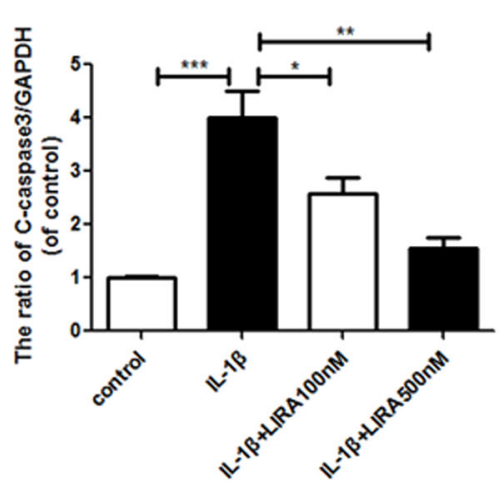

Fig. 2 Activation of GLP-1R by liraglutide decreases apoptosis in chondrocytes. a CCK-8 assays of chondrocytes treated with various concentrations of liraglutide for $24 \mathrm{~h}$ as shown above. $\mathbf{b}$ CCK-8 assays of liragltide-pretreated chondrocytes stimulated by IL-1 $\beta$. c Chondrocytes were pretreated with liraglutide and then IL-1 $\beta$ and imaged by phase-contrast microscopy (20X). $\mathbf{d}-\mathbf{g}$ Representative western blots and quantification data of $\mathrm{Bax}, \mathrm{BCl}-2$ and cleaved-caspase 3 in each group. Columns represent mean \pm SD, Significant differences between the treatment and control groups are indicated as ${ }^{*} P<0.05,{ }^{* *} P<0.01,{ }^{* * *} P<0.001, n=5$ 


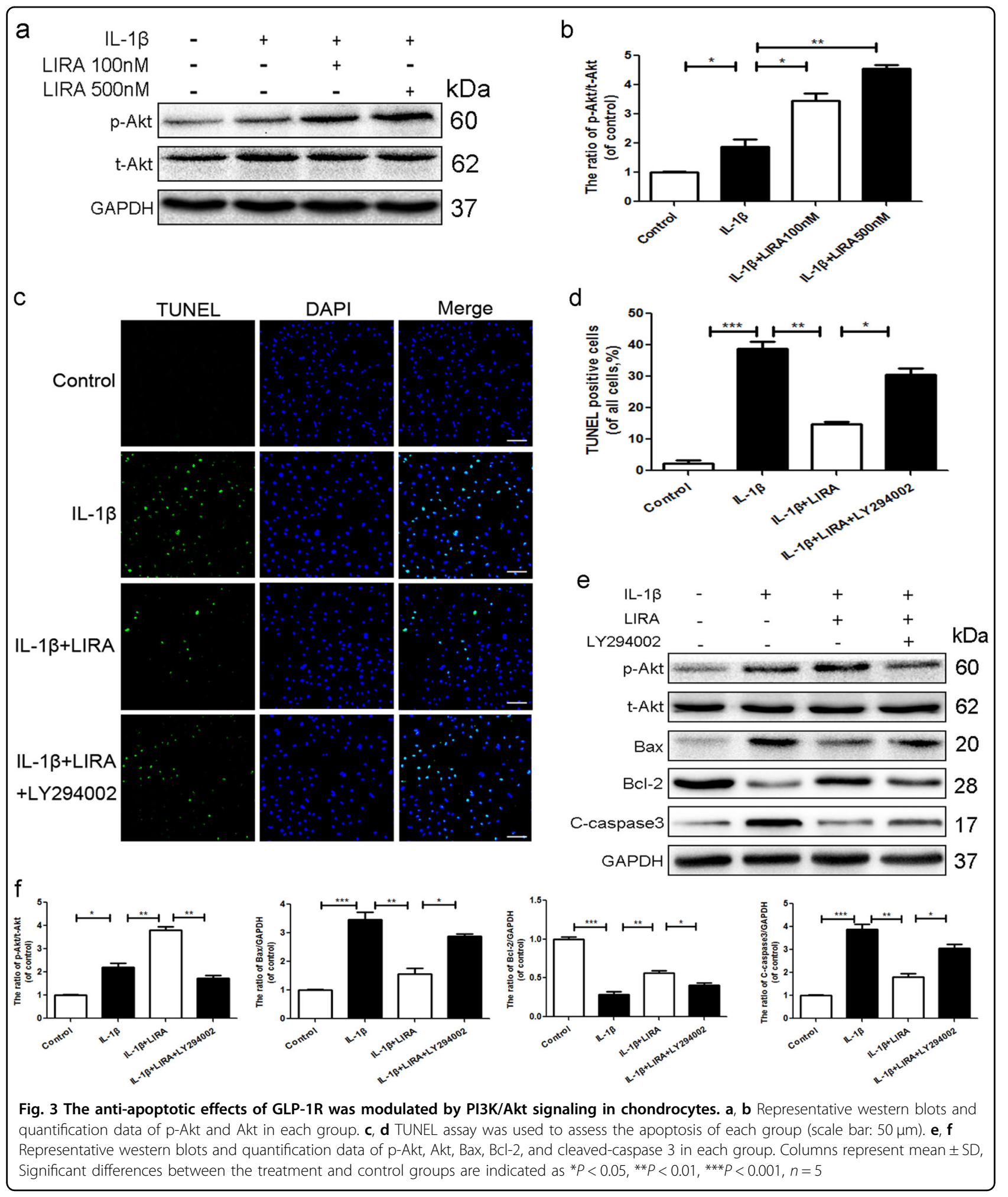

GLP-1R may relate ER stress and NF-KB pathway in chondrocytes

Several studies have noted the inflammation-related changes in the OA cartilage. Then we test the protein levels of $\mathrm{p}-\mathrm{I} \kappa \mathrm{B} \alpha, \mathrm{I} \kappa \mathrm{B} \alpha$, and NF- $\kappa \mathrm{B}$ (p65) under the TG treatment using the western blot. I $\mathrm{K} \alpha$ was an upstream target of NF- $\mathrm{KB}$, its phosphorylation and degradation contribute to the activation of the NF- $\mathrm{BB}$ pathway. 


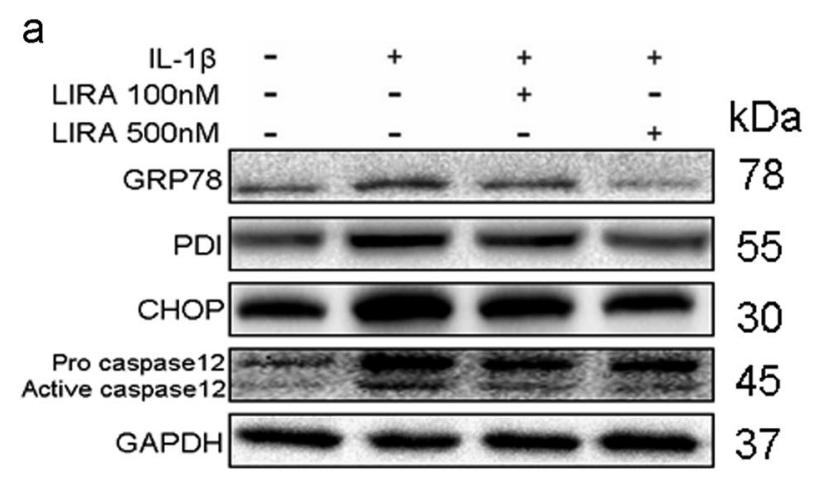

b

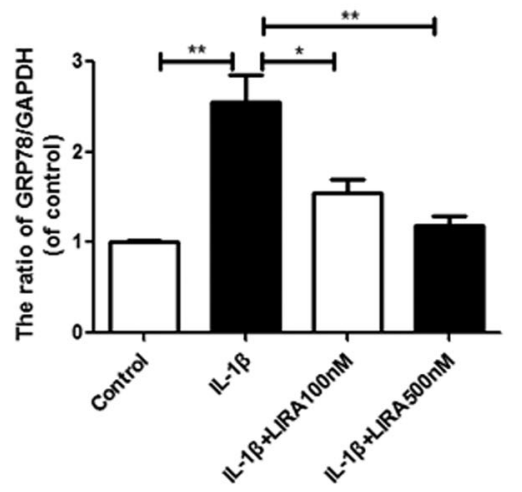

C

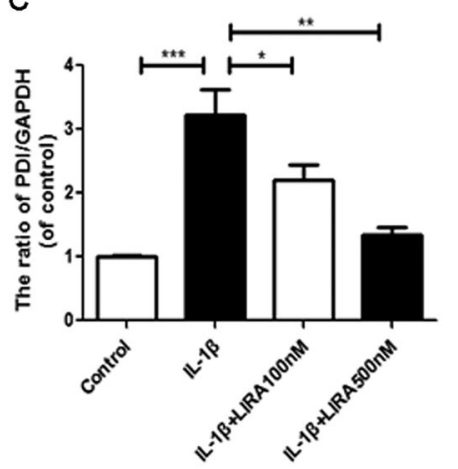

d

f
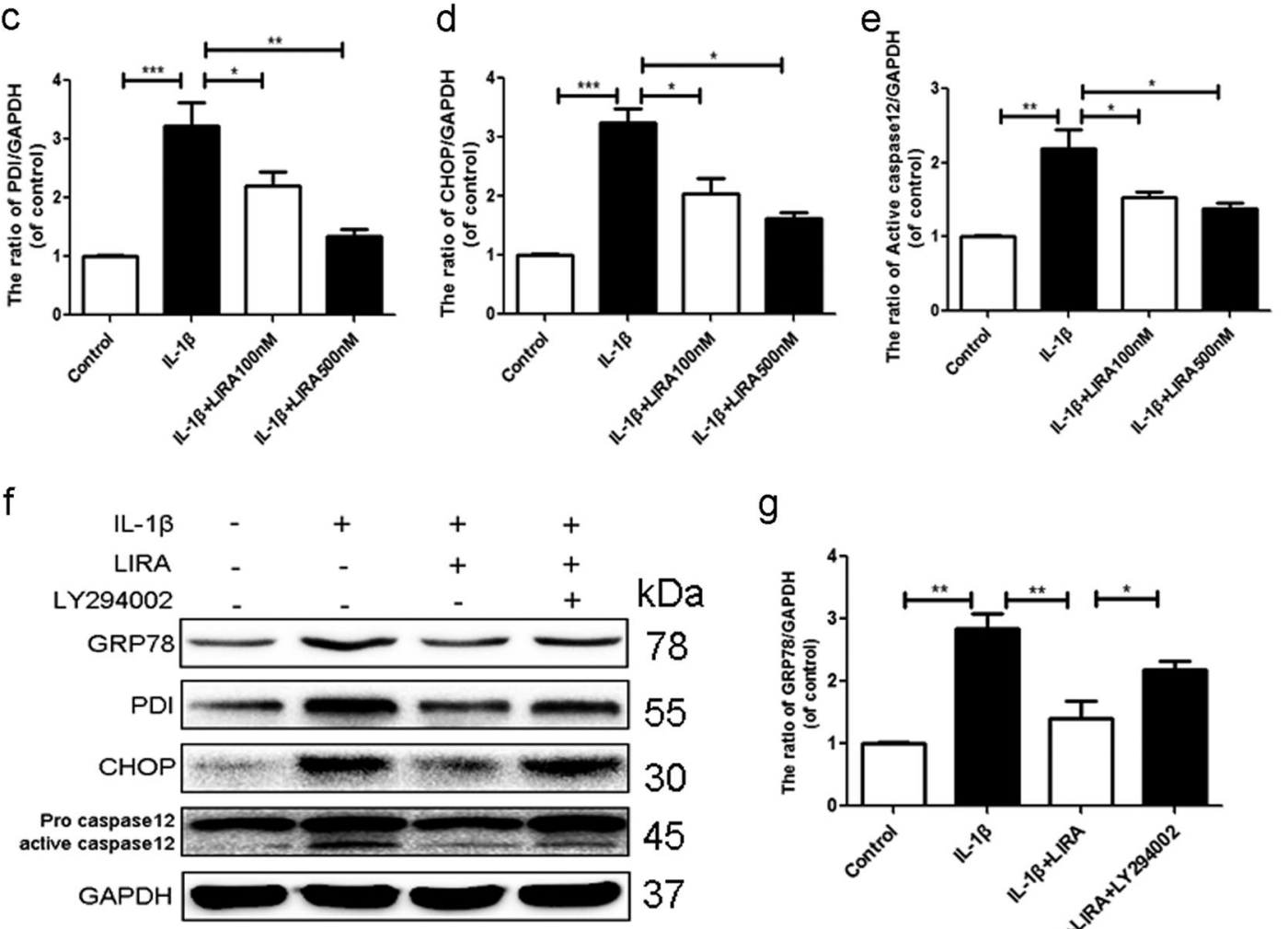

g

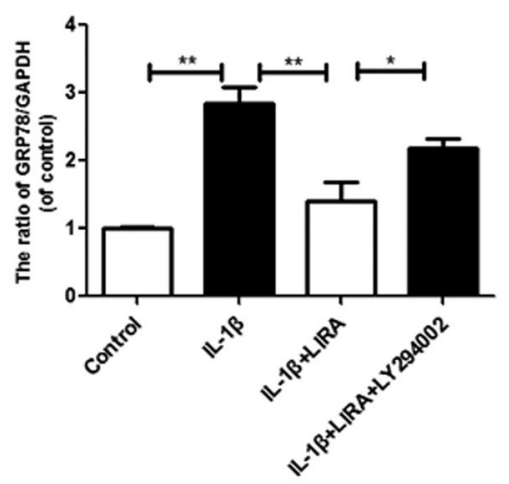

h
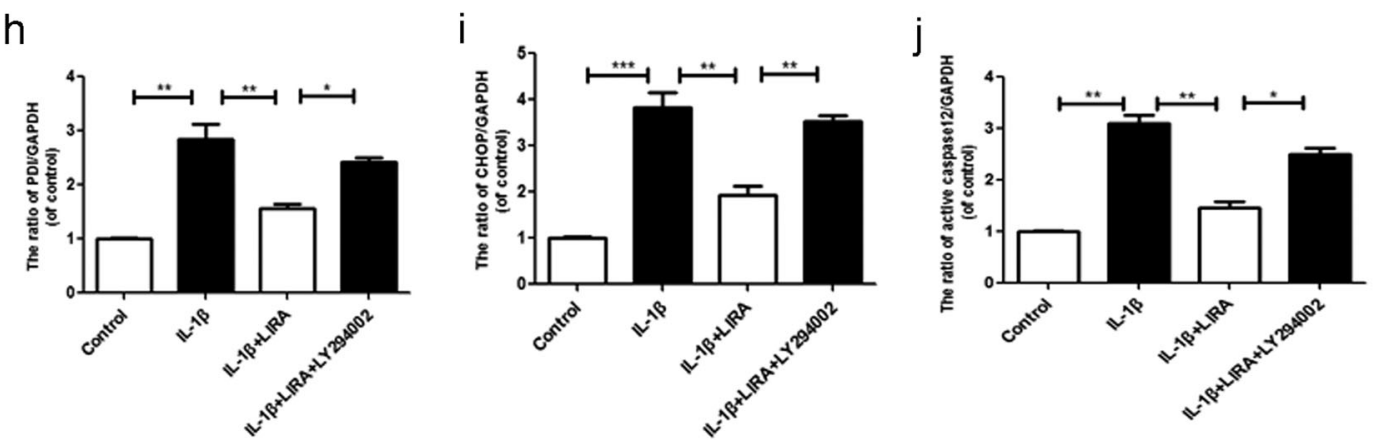

Fig. 4 Activation of GLP-1R inhibits ER stress via activation of PI3K/Akt signaling in chondrocytes. a-e Representative western blots and quantification data of GRP78, PDI, caspase 12 and CHOP in each group. $\mathbf{f}-\mathbf{j}$ Representative western blots and quantification data of GRP78, PDI, caspase 12 and $\mathrm{CHOP}$ in each group. Columns represent mean \pm SD, Significant differences between the treatment and control groups are indicated as ${ }^{*} P<0.05,{ }^{* *} P<0.01,{ }^{* * *} P<0.001, n=5$ 


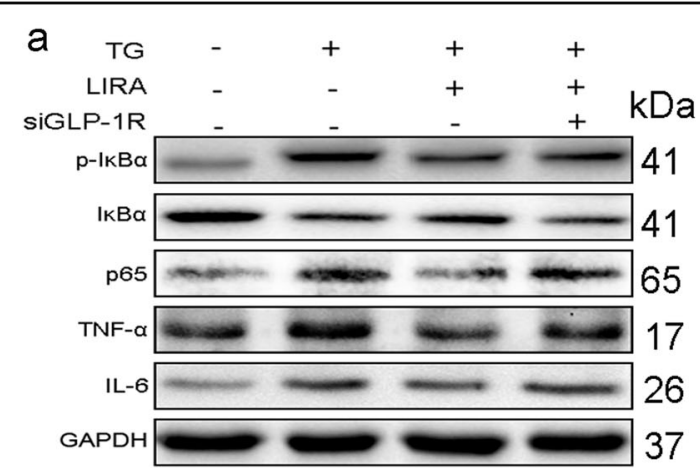

C

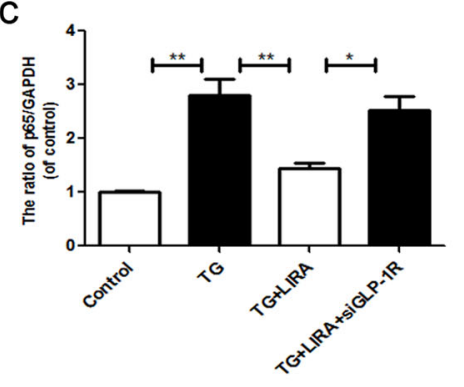

f

Control
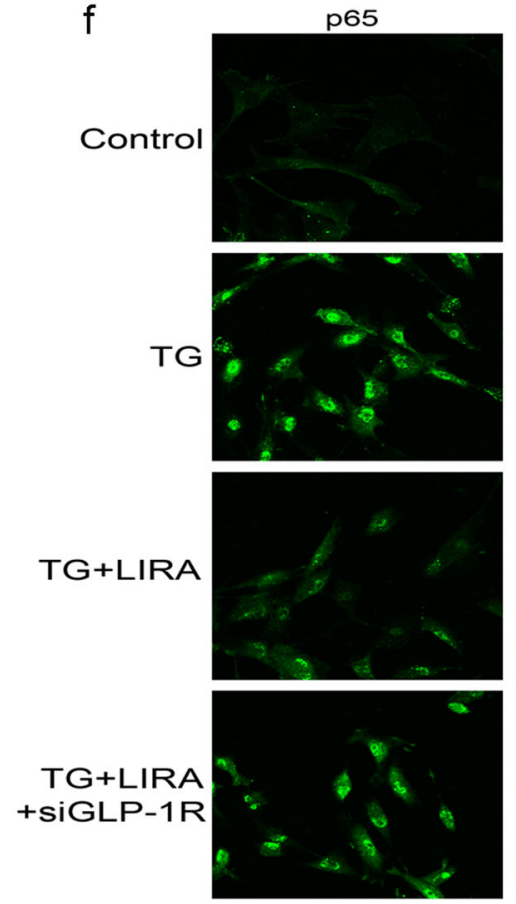

d
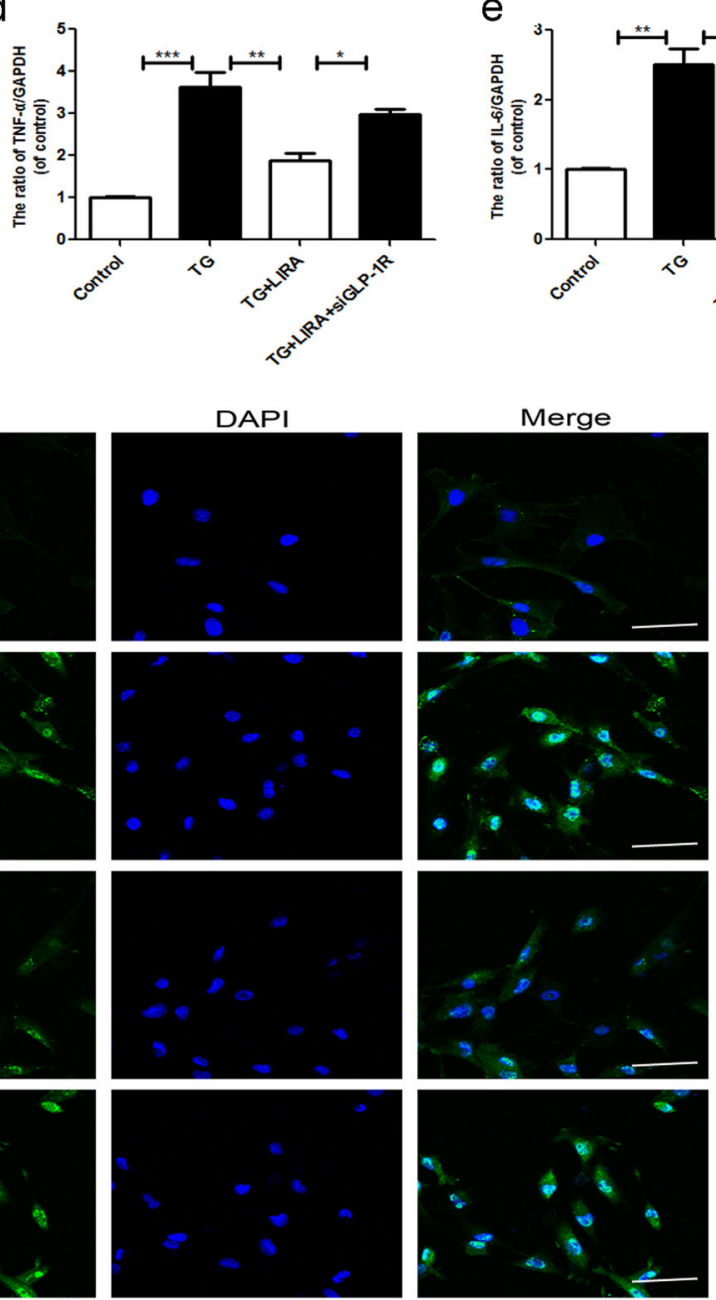

Fig. 5 GLP-1R may relate ER stress and NF-KB pathway in chondrocytes. a-f Representative western blots and quantification data of p65, p-IKBa and IKBa, IL-6, TNF-a in each group. $\mathbf{g}$ Immunofluorescence staining of P65 proteins (green) and nucleus (blue) was labeled with DAPI (scale bar: 50 $\mu \mathrm{m})$. Columns represent mean $\pm S D$, Significant differences between the treatment and control groups are indicated as ${ }^{*} P<0.05,{ }^{* *} P<0.01,{ }^{* * *} P<$ $0.001, n=5$

Compare to the control group, higher expression of $\mathrm{p} 65$, IL-6, TNF- $\alpha$, and ratio of $\mathrm{p}-\mathrm{I} \kappa \mathrm{B} \alpha / \mathrm{I \kappa} \mathrm{B} \alpha$ were noted in TG group indicating the activation of NF- $\mathrm{kB}$ pathway and associated inflammatory response may relate to ER stress. However, liraglutide treatment inhibited the activation of
NF- $\kappa B$ and subsequent inflammatory response, which was reversed by GLP-1R siRNA (Fig. 5a-e). NF-kB nuclear translocation triggers release of inflammatory mediators. Immunostaining assays showed that activating GLP-1R significantly attenuated TG induced nuclear translocation 
a

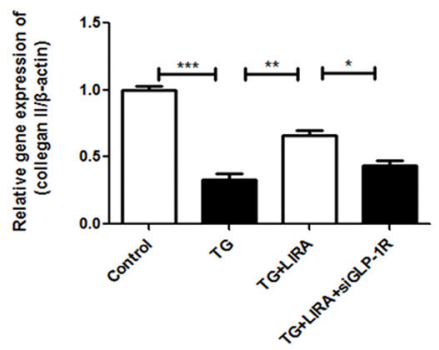

C
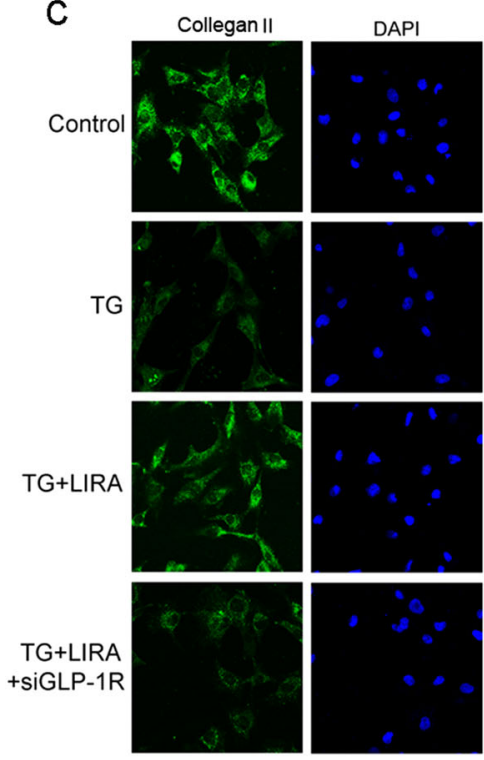

Merge
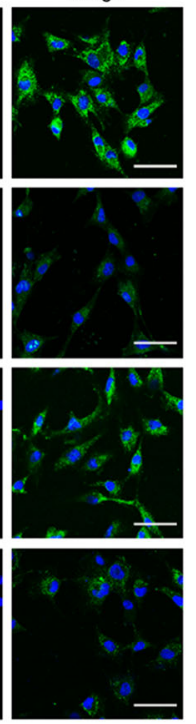

b

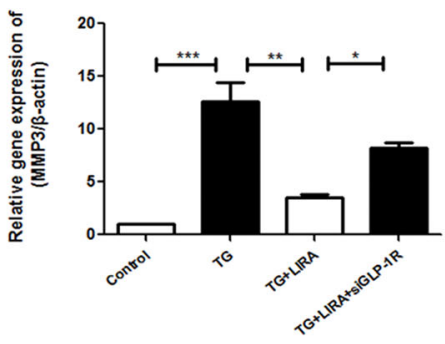

d
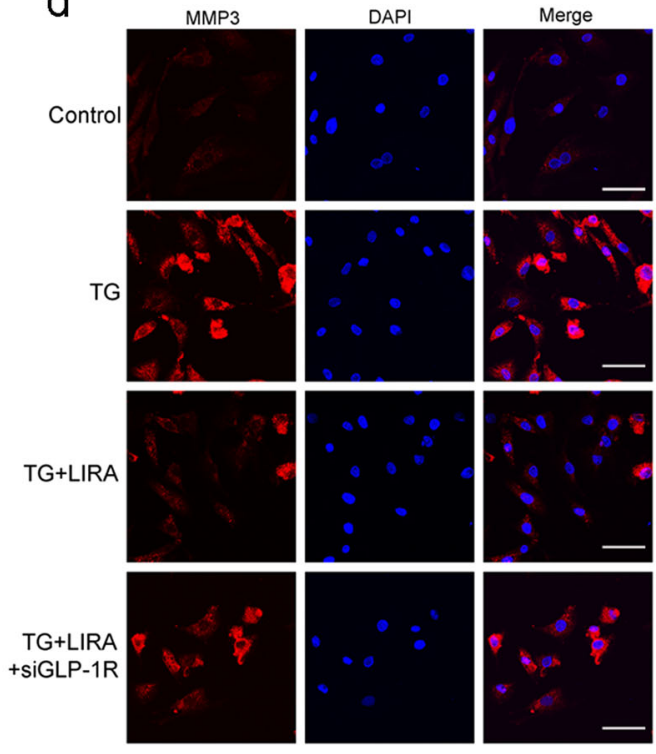

Fig. 6 GLP-1R activation decreased the ECM catabolic activity in TG-treated chondrocytes. $\mathbf{a}, \mathbf{b}$ The relative mRNA expression of MMP-3 and collegan-II in each group. c, d Immunofluorescence staining of MMP-3 and collegan-II proteins in each group (scale bar: $50 \mu m$ ). Columns represent mean \pm SD, Significant differences between the treatment and control groups are indicated as ${ }^{*} P<0.05,{ }^{* *} P<0.01,{ }^{* *} P<0.001, n=5$

of NF- $\mathrm{BB}$, which was also abolished by GLP-1R siRNA (Fig. 5f). Together, these results showed that GLP-1R may play an essential role in ER stress-induced inflammatory pathways.

\section{GLP-1R activation decreased the ECM catabolic activity in TG-treated chondrocytes}

We tested the effect of liraglutide on TG-induced metabolic activity of ECM in chondrocytes using PCR and immunofluorescence assay, including major ECM protein collegan-II and ECM degrading protein MMP-3. As shown in Fig. 6a, b, TG treatment markedly reduced mRNA expression of collegan II, and upregulated the mRNA levels of MMP3. However, liraglutide attenuated the ECM catabolic activity, and the effect of GLP-1R were significantly abolished by GLP-1R siRNA. And immunofluorescence evaluation of collagen-II and MMP3 protein expression keeps consistent with the mRNA results (Fig. 6c, d), indicating GLP-1R may regulate the ECM metabolic activity in chondrocyte.
Liraglutide treatment ameliorated chondrocytes apoptosis and cartilage degeneration in rat $O A$ model

The histopathological change of the matrix layer and articular structure was observed by $\mathrm{HE}$, Safranin $\mathrm{O}$ staining and OARSI scores. In the OA group, morphological structure of articular cartilage showed significant destruction, including cartilage erosion, proteoglycan and cellular loss, compared with control group. Importantly treatment with liraglutide significantly reduced the severity of cartilage degeneration in the OA cartilages, which was consistent with OARSI scores (Fig. $7 \mathrm{a}-\mathrm{c}$ ). And immunohistochemical staining and corresponding quantification showed that liraglutide can greatly decreased the cytoplasmic $\mathrm{CHOP}$ and caspase 3 positive in rat articular cartilage compared with OA model (Fig. 7d-g), which confirmed the results of our in vitro studies. Our data showed that GLP-1R agonist has a significant protective effect on vivo. 


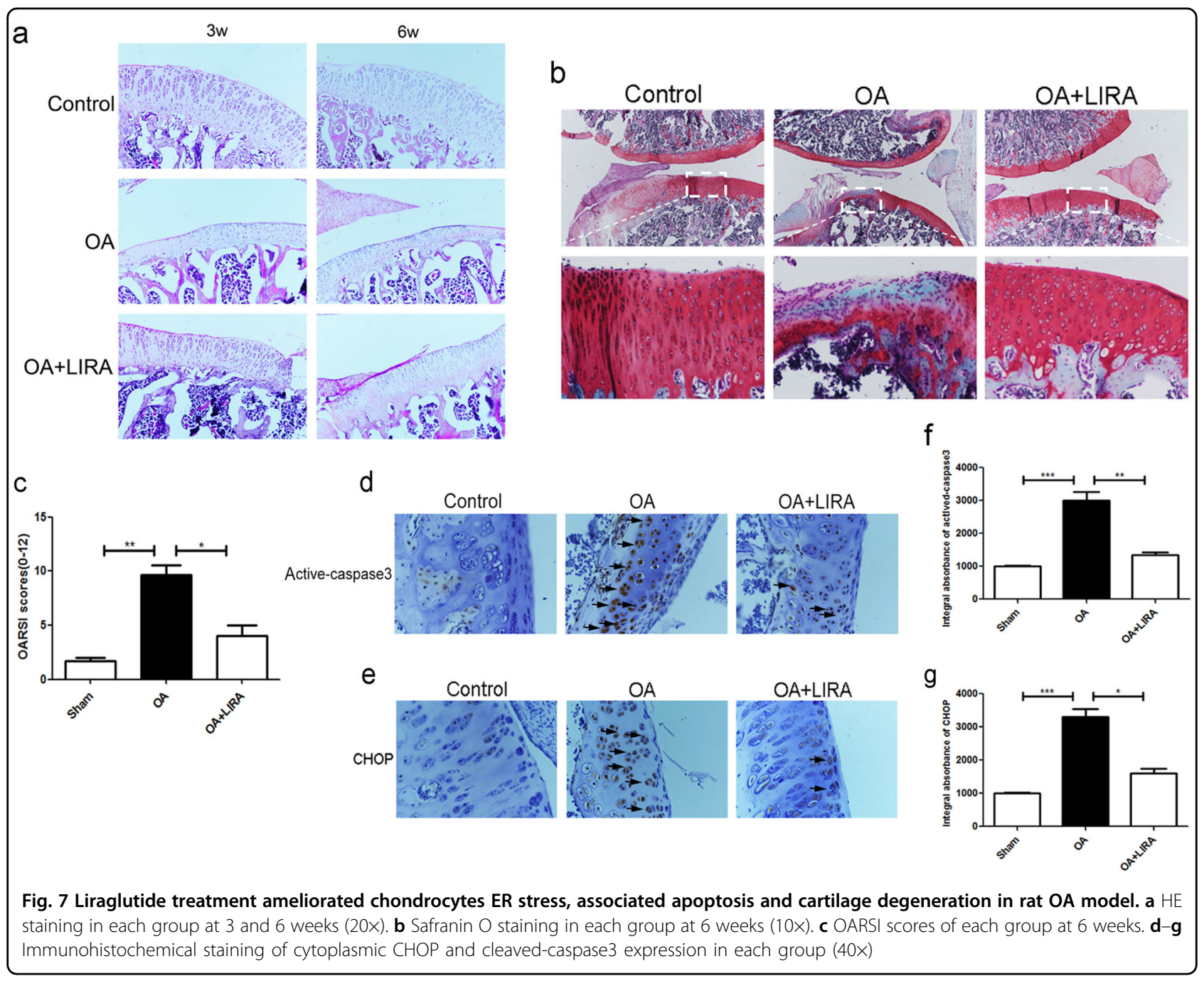

\section{Discussion}

The process of OA has been attributed to several processes, such as aging and excessive mechanical loading, which trigger a series of molecular events leading to cell death and structural damage in articular cartilage, particularly inflammation ${ }^{23-25}$. The degradation of articular cartilage is mainly due to dysregulation of chondrocyte apoptosis and excessive catabolism of $\mathrm{ECM}^{26-28}$. Several studies reported that chondrocyte apoptosis contributes to cellular changes in cartilage and loss of articular cartilage $^{29,30}$. Many important mediators, such as IL-1 $\beta$ and TNF- $\alpha$, recruit death-inducing signaling complexes via binding with respective ligands, and subsequently activate the caspase signaling pathway, ultimately leading to internucleosomal DNA fragmentation ${ }^{31,32}$. Inflammation plays a vital role in ECM metabolic processes, and increasing evidence suggests that inflammation-mediated overexpression of MMPs results in a progressive matrix catabolic process ${ }^{5,33}$
Specific ligands bind to GLP-1R and subsequently mediate multiple biological processes, which occur in many tissues, such as the brain, heart, and pancreatic islets $^{34-37}$. Nevertheless, little is known about the role of GLP-1R in OA. Increasing evidence indicates that GLP$1 \mathrm{R}$ has extensive links with anti-apoptotic effects. GLP-1 analogs reportedly protect cholangiocytes against apoptosis by preventing mitochondrial translocation of Bax and caspase- 3 activation, in vitro and in vivo ${ }^{38}$. It has been demonstrated that the protective effects of GLP-1R could be mediated by PI3K/Akt signaling ${ }^{21,22}$. The PI3K/Akt pathway plays pivotal role in growth and survival of many biological processes, such as ischemia injury and degenerative diseases. Phosphorylated Akt reduces the proapoptotic protein Bad, and attenuates apoptotic activity after brain injury ${ }^{39}$. Akt also inactivates pro-caspase-9, and causes caspase 9 dephosphorylation, decreasing the level of apoptosis ${ }^{40}$. It has been reported that GLP-1R activation inhibits hypoxia/reoxygenation damage in cardiac microvascular endothelial cells via the PI3K/Akt 
signaling ${ }^{21}$. This work first noted the decreasing expression of GLP-1R in degenerative cartilage, indicating the dysfunction of GLP-1R pathway during the process of OA. And to further explore whether the activation of GLP-1R exerted a protective effect on chondrocytes, the GLP-1R agonist liraglutide was used in IL-1 $\beta$-stimulated chondrocytes. Activation of GLP-1R markedly reduced the levels of pro-apoptotic Bax and cleaved-caspase3, and increased anti-apoptotic Bcl-2 in IL-1 $\beta$-induced chondrocytes, indicating that GLP-1R may be a potential target for the treatment of OA. To further explore the potential mechanism of GLP-1R, LY294002, a specific PI3K/Akt inhibitor was used to treat chondrocytes combined with liraglutide treatment. Interestingly, LY294002 abolished the anti-apoptotic effects of GLP-1R, indicating that PI3K/Akt signaling may involve in the protective effects of GLP-1R.

ER stress is closely related to apoptosis in OA. During the process of ER stress, UPR sensors separate from chaperone proteins, such as GRP78 and PDI, and then become phosphorylated, which activates $\mathrm{CHOP}$ and apoptotic proteins, such as caspase-12, to induce apoptosis $^{41}$. Evidence shows that there is less apoptosis and cartilage degeneration in arthrosis of CHOP knockout mice $^{42}$. Moreover, it has been shown that a GLP-1R agonist attenuated ER stress by downregulating ATF-4, a biomarker of ER stress, and inhibited apoptosis in INS-1 $\beta$-cells, which could improve cell survival ${ }^{43}$. Additionally, exenatide 4, a GLP-1R agonist, alleviated lipid-induced ER stress and improved hepatic steatosis in high-fat diet -fed mice via sirtuin 1 (SIRT1) ${ }^{44}$. Our data showed that pretreatment with a GLP-1R agonist alleviated the expression of ER stress-related proteins, such as PDI, GRP78, CHOP, and caspase-12, which increase in response to ER stress and are considered to be mediators of apoptotic pathways $^{45,46}$, which were partial reversed by LY294002. These results indicated that the protective effect of the GLP-1R agonist may be attributable to the inhibition of ER stress in chondrocytes. And inhibiting PI3K/Akt pathway abolished the protective effects of GLP-1R by attenuating ER stress. Moreover, to confirm the protective role of agonist-activated GLP-1R, siRNA targeted to GLP-1R and TG, an inducer of ER stress, were used to treat chondrocytes. Treatment with liraglutide reduced the TGinduced accumulation of these ER stress-related proteins and subsequently decreased ER stress related-apoptosis in chondrocytes. These effects were abolished by GLP-1R siRNA treatment, further demonstrating that GLP-1R may exert a protective effect in chondrocytes against ERassociated apoptosis in OA. Additionally, in vivo experiments also demonstrated that treatment with liraglutide attenuated ER stress related-apoptosis and the destruction of articular cartilage compared with the OA group. These results indicated a therapeutic effect of the GLP-1R agonist in the rat OA model and were consistent with the results in vitro.

Inflammatory responses are a vital contributor to ECM degradation in $\mathrm{OA}^{5,47}$. Various inflammatory factors can trigger the phosphorylation and degradation of IкB and translocate NF- $\mathrm{KB}$ into the nucleus, facilitating inflammatory protein synthesis and pro-inflammatory molecule release (IL-6, TNF- $\alpha$ ), leading to cartilage degeneration ${ }^{48-}$ ${ }^{50}$. Previous studies showed that NF-kB (p65) knockdown could significantly reduce the expression of MMPs and inflammatory factors in chondrocytes that were treated with IL- $1 \beta$ and TNF- $\alpha^{51}$. Moreover, other studies reported that the GLP-1R signaling pathway is antiinflammatory via regulation of NF-KB signaling and subsequent promotion of cellular function in different cells, including pancreatic $\beta$-cells, macrophages, and endothelial cells ${ }^{52-55}$.

Recent studies demonstrated that ER stress is involved in inflammatory responses ${ }^{5-58}$. ER-resident IRE1 $\alpha$ is required to activate NF- $\mathrm{KB}$ translation in response to ER stress through TRAF2-mediated formation of IRE1 $\alpha$ and IKK complex. Inhibition of NF- $\kappa B$ suppressed ER stressinduced cell death in MCF-7 cells ${ }^{59}$. Therefore, we assumed that the GLP-1R agonist inhibited ER stress and subsequently the inflammatory response during the process of ECM degradation. These results showed that TGinduced ER stress triggered nuclear translation of NF- $\mathrm{kB}$, contributing to pro-inflammatory cytokine transcription and subsequently promoting an ECM catabolic process. Furthermore, activation of GLP-1R significantly inhibited the ER stress-induced nuclear translation of NF- $\mathrm{KB}$ and reduced expression levels of inflammatory molecules in TG-induced chondrocytes. Moreover, liraglutide prevented the degradation of ECM components by downregulating matrix-degrading enzymes, such as MMP-3, suggesting that the GLP-1R agonist modulated the process of reducing the ER stress-related inflammatory response and alleviated the ECM catabolic process. Moreover, the anti-inflammatory effect of liraglutide via regulating ER stress was abolished by GLP-1R siRNA.

In conclusion, this study provides mechanistic evidence to demonstrate the anti-apoptotic and anti-inflammatory effects of GLP-1R activation in chondrocytes, in vivo and in vitro. Regulation of PI3K/Akt/ER stress was closely involved in the protective effects of GLP-1R (Fig. 8). Overall, these results demonstrate that GLP-1R may be a novel target for the treatment of OA, particularly in patients with diabetes mellitus.

\section{Materials and methods \\ Chemicals and reagents}

Liraglutide was obtained from Novo Nordisk (Princeton, NJ, USA). The IL- $1 \beta$ was from Peprotech. Antibodies against GRP78, PDI, ІкB- $\alpha, \mathrm{p}-\mathrm{I \kappa B}-\alpha$, and collagen II were 


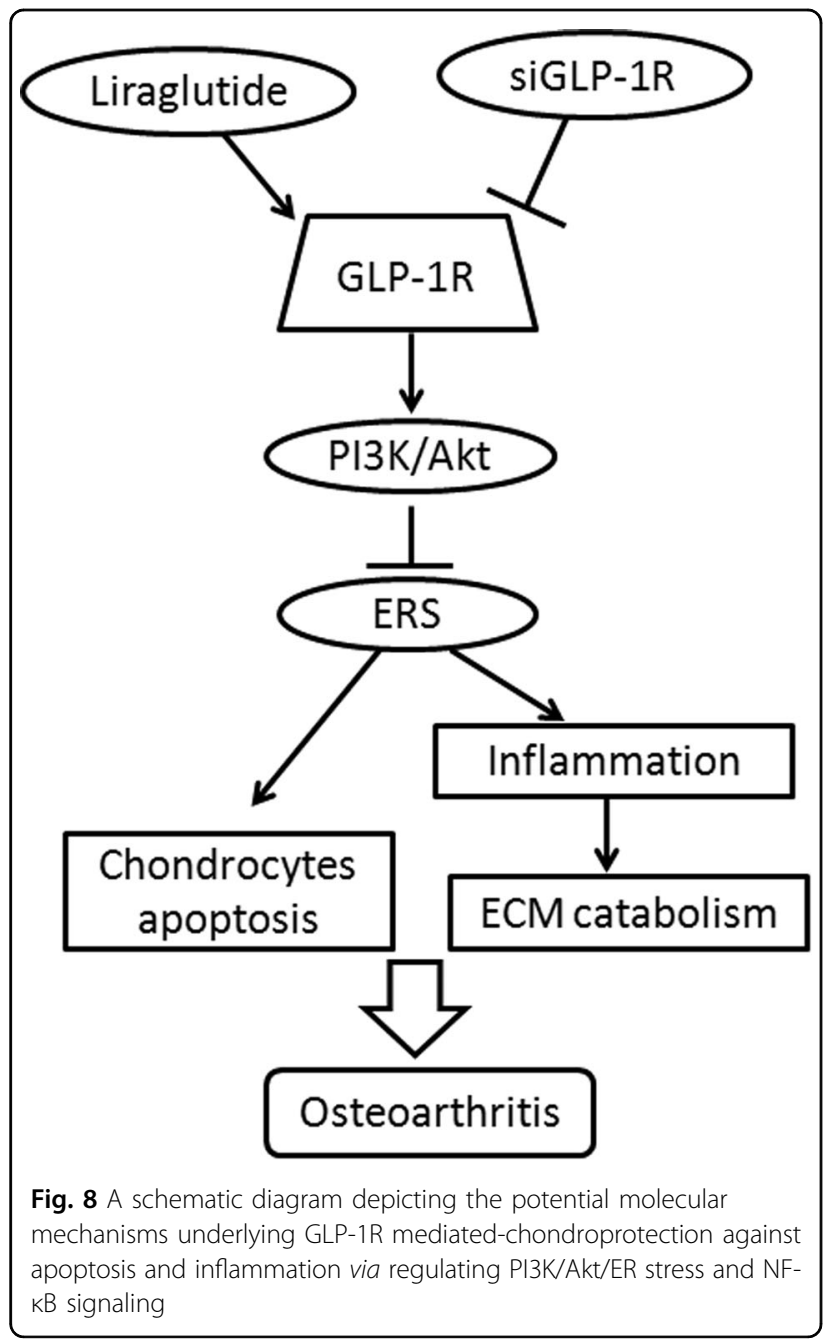

obtained from Abcam (Cambridge, MA, USA). Antibodies against CHOP, p65, cleaved-caspase3 and specific PI3K inhibitor (LY294002) were obtained from Cell Signaling Technology (Beverly, MA, USA). Antibodies against GLP1R, MMP3, IL-6, TNF- $\alpha$, Bax and Bcl-2 were obtained from Santa Cruz Biotechnology (Santa Cruz, CA, USA). Thapsigargin (TG) and other reagents were obtained from Sigma unless noted otherwise.

\section{Cell isolation and culture}

Chondrocytes were extracted using the bilateral knee joints of SD rats (Animal Center of the Chinese Academy of Sciences, Shanghai, China.). All surgical interventions, and postoperative animal care conformed to the Animal Care and Use Committee of Wenzhou Medical University. All rats were housed under specific pathogen-free (SPF) laboratory conditions. Knee-joint cartilage was sectioned into pieces and incubated with $0.25 \%$ TrypsinEDTA for $30 \mathrm{~min}$, and placed in $0.2 \%$ type II collagenase for $4 \mathrm{~h}$. After resuspension and filtration, chondrocyts were cultured in DMEM, which contained $10 \%$ fetal bovine serum (FBS) and antibiotics (1\% penicillin and streptomycin $)^{60,61}$. Morphologically, chondrocytes appear rounded and containing large amounts of aggrecan, a large proteoglycan, and collegan-II, with smaller amounts of other collagens. And Cell fluorescence results of collegan-II showed that most of the cells highly expressed collegan-II (Supplementary Figure S4). Chondrocytes between passage 0 and passage 2 were used for all experiments.

\section{Cell viability assay}

Cell viability was tested using CCK-8 kit (Dojindo, Japan) according to the its protocol. Briefly, cells were plated in 96-well plates. After reaching a confluence of $80-90 \%$, the cells were treated with or without different concentrations of liraglutide (as described) for $2 \mathrm{~h}$, followed by stimulation with or without IL- $1 \beta 10 \mathrm{ng} / \mathrm{ml}$ for $24 \mathrm{~h}$. Then, CCK-8 dye $(10 \mu \mathrm{L})$ was added in each well, followed by incubation for $2 \mathrm{~h}$. The absorbance was tested using a 96-well plate reader at $450 \mathrm{~nm}$ (Thermo, Rockford, IL, USA).

\section{TUNEL method}

The TUNEL method was used to assess apoptosis in each group of chondrocytes. After fixation with $4 \%$ paraformaldehyde (PFA) for $1 \mathrm{~h}$, chondrocytes were incubated with $3 \%$ hydrogen peroxide $\left(\mathrm{H}_{2} \mathrm{O}_{2}\right)$ for $10 \mathrm{~min}$ and $0.1 \%$ Triton $\mathrm{X}-100$ for $5 \mathrm{~min}$. Then, apoptotic chondrocytes were stained using an in situ Cell Death Detection Kit and subsequently stained with DAPI for $7 \mathrm{~min}$. Images were captured by a Nikon ECLIPSE Ti microscope (Japan).

\section{Immunofluorescence staining}

Immunofluorescence assays were used to assess the protein levels of cleaved-caspase3, p65, MMP3 and collagen II. The chondrocytes were planted on glass coverslips in 6-well plates. After treatment, samples were fixed with $4 \%$ PFA for $1 \mathrm{~h}$, and permeabilised by $0.5 \%$ Triton X100 for 10 min. After block with $1 \%$ bovine serum albumin (BSA) at $4{ }^{\circ} \mathrm{C}$ for $30 \mathrm{~min}$, the primary antibodies were incubated overnight. The next day, samples were incubated with Alexa Fluor 488-conjugated anti-IgG secondary antibodies and Alexa Fluor 647-conjugated anti-IgG for $1 \mathrm{~h}$ and stained with DAPI for $7 \mathrm{~min}$. Samples were observed by a Nikon ECLIPSE Ti microscope (Japan).

\section{Western blot}

Total protein of chondrocytes was extracted using RIPA buffer with protease and phosphatase inhibitors. Bicinchoninic acid (BCA) reagents (Thermo, Rockford, IL, USA) were used to measure protein concentrations. Total proteins were loaded onto the SDS-PAGE gels (10-12\%) 
Table 1 Primers of targeted genes

\begin{tabular}{lll}
\hline Gene & Forward & Reverse \\
\hline MMP-3 & GCTGTTITGAAGAATTGGGTTC & GCACAGGCAGGAGAAAACGA \\
Collagen-II & CTCATCCAGGGCTCCAATGA & CCATGGGTGCAATGTCAACA \\
\hline
\end{tabular}

and then transferred to PVDF membranes. Following blocked in 5\% BSA for $1 \mathrm{~h}$, the bands were incubated with respective primary antibodies overnight and secondary antibody for $1 \mathrm{~h}$. Finally, the blot signals were visualized using a ChemiDoc ${ }^{\mathrm{TM}}$ XRS + Imaging System (Bio-Rad) from at least three independent experiments.

\section{Small interfering RNA (siRNA)}

GLP-1R expression in chondrocytes was silenced by transfection of siRNA. Chondrocytes were transfected with GLP-1R siRNA (GeneChem, Shanghai, China) using lipofectamine 2000 (Life Technologies, Carlsbad, CA, USA) according to the instruction. Six hours after transfection, medium was switched to medium containing $5 \%$ FBS for $24 \mathrm{~h}$. Then the chondrocytes were treated as described for further experiments.

\section{RT-PCR}

After treatments, total RNA of cells was isolated using the TRIzol method. After synthesis using the PrimeScriptRT reagent kit from total RNA, the cDNA was amplificated by the PrimeScript-RT reagent kit and SYBR Premix Ex Taq (Sangon). Forward and reverse primer sequences of MMP-3 and collagen-II are showed in Table 1. The expression of each gene was measured by the DDCt method, as mentioned previously ${ }^{62}$.

\section{OA model}

Fifteen male SD rats (200-250 g) were randomly assigned into three groups: Sham, OA, and OA + liraglutide group. Briefly, after anesthesia with chloral hydrate $(10 \%)$, the right knees of rats were prepared for aseptic surgery. The OA model was cut off anterior cruciate ligament (ACL) transection combined with medial menisci resection on the right knee joint ${ }^{63}$. The rats in the normal group received the same incision without the resection of ACL and meniscus. After surgery, all the rats in the OA + liraglutide group administered at $50 \mu \mathrm{g} / \mathrm{kg} /$ day subcutaneously (s.c.) until the rats were sacrificed. Equal saline injections were administered for the sham group and OA group.

\section{Histological analysis}

Cartilaginous tissue was surgically excised from the right knee joints of the rats and fixed immediately in $4 \%$ PFA for $48 \mathrm{~h}$ and decalcified for 14 days. Then, the tissues were dehydrated and subsequently embedded in paraffin wax. The tissue sections $(5 \mu \mathrm{m})$ covering whole joints were cut for HE staining and Safranin O staining to assess cartilage destruction. The images were captured using a light microscope, and the level of cartilage degeneration was measured by the Osteoarthritis Research Society International (OARSI) scores ${ }^{64}$.

\section{Immunohistochemical analysis}

The levels of GLP-1R, cleaved-caspase 3 and CHOP were evaluated in each group using immunohistochemical staining. Briefly, the sections were deparaffinised and rehydrated, following blocked with $3 \% \mathrm{H}_{2} \mathrm{O}_{2}$ for $10 \mathrm{~min}$ and $5 \% \mathrm{BSA}$ for $30 \mathrm{~min}$. And the tissue sections were incubated with primary antibodies overnight and HRPconjugated secondary antibody for $1 \mathrm{~h}$, following stained with haematoxylin for $8 \mathrm{~min}$. Images were captured using a light microscope. Images were analysed by Image-Pro Plus software, version 6.0 (Media Cybernetics, Rockville, MD, USA), and the integral absorbance values were used to measure the level of protein. At least three sections from each specimen were used to measure the level of protein.

\section{Statistical analysis}

The results are presented as mean \pm SD for each group from at least three independent experiments. Statistical comparisons of the data between each group were conducted using the Graphpad Prism software (one-way analysis of variance (ANOVA) followed by Tukey's test). $P$ values $<0.05$ were considered statistically significant.

\section{Acknowledgements}

This work is supported by National Nature Foundation of China (81371988), Zhejiang Provincial Health Department Foundation of China (2016KYB196 and LY15H060008); Zhejiang Traditional Chinese Medicine Bureau Foundation of China (2016ZA140).

\section{Author details}

${ }^{1}$ Department of Orthopaedics, The Second Affiliated Hospital and Yuying Children's Hospital of Wenzhou Medical University, Wenzhou 325027, People's Republic of China. ${ }^{2}$ School of Pharmaceutical Sciences, Key Laboratory of Biotechnology and Pharmaceutical Engineering, Wenzhou Medical University, Wenzhou 325027 Zhejiang, China. ${ }^{3}$ The first clinical college, Wenzhou Medical University, 325027 Wenzhou, People's Republic of China. ${ }^{4}$ Biological science academy, Wenzhou university, 325027 Wenzhou, Zhejiang, China

\section{Conflict of interest}

The authors declare that they have no conflict of interest. 


\section{Publisher's note}

Springer Nature remains neutral with regard to jurisdictional claims in published maps and institutional affiliations.

Supplementary Information accompanies this paper at (https://doi.org/ 10.1038/s41419-017-0217-y).

Received: 20 August 2017 Revised: 11 November 2017 Accepted: 7 December 2017

Published online: 12 February 2018

\section{References}

1. Cross, M. et al. The global burden of hip and knee osteoarthritis: estimates from the global burden of disease 2010 study. Ann. Rheum. Dis. 73, 1323-1330 (2014).

2. Silverwood, V. et al. Current evidence on risk factors for knee osteoarthritis in older adults: a systematic review and meta-analysis. Osteoarthr. Cartil. 23 507-515 (2015)

3. Heep, H., Hilken, G., Hofmeister, S. \& Wedemeyer, C. Osteoarthitis of leptindeficient ob/ob mice in response to biomechanical loading in micro-CT. Int. J. Biol. Sci. 5, 265-275 (2009).

4. Loeser, R. F., Goldring, S. R., Scanzello, C. R. \& Goldring, M. B. Osteoarthritis: a disease of the joint as an organ. Arthritis Rheum. 64, 1697-1707 (2012).

5. Kapoor, M., Martel-Pelletier, J., Lajeunesse, D., Pelletier, J. P. \& Fahmi, H. Role of proinflammatory cytokines in the pathophysiology of osteoarthritis. Nat. Rev. Rheumatol. 7, 33-42 (2011).

6. Goldring, M. B. et al. Roles of inflammatory and anabolic cytokines in cartilage metabolism: signals and multiple effectors converge upon Mmp-13 regulation in osteoarthritis. Eur. Cells Mater. 21, 202-220 (2011).

7. Chen, D. et al. 1,25-Dihydroxyvitamin D3 activates MMP13 gene expression in chondrocytes through p38 MARK pathway. Int. J. Biol. Sci. 9, 649-655 (2013).

8. Latourte, A. et al. Systemic inhibition of IL-6/Stat3 signalling protects against experimental osteoarthritis. Ann. Rheum. Dis. 76, 748-755 (2016).

9. Hotamisligil, G. S. Endoplasmic reticulum stress and the inflammatory basis of metabolic disease. Cell 140, 900-917 (2010).

10. Claudio, N., Dalet, A., Gatti, E. \& Pierre, P. Mapping the crossroads of immune activation and cellular stress response pathways. Embo J. 32, 1214-1224 (2013).

11. Garg, A. D. et al. ER stress-induced inflammation: does it aid or impede disease progression? Trends Mol. Med. 18, 589-598 (2012).

12. Janicke, R. U., Sprengart, M. L., Wati, M. R. \& Porter, A. G. Caspase-3 is required for DNA fragmentation and morphological changes associated with apoptosis. J. Biol. Chem. 273, 9357-9360 (1998).

13. Guo, F. J. et al. ATF6 upregulates XBP1S and inhibits ER stress-mediated apoptosis in osteoarthritis cartilage. Cell. Signal. 26, 332-342 (2014).

14. Husa, M., Petursson, F., Lotz, M., Terkeltaub, R. \& Liu-Bryan, R. C/EBP homologous protein drives pro-catabolic responses in chondrocytes. Arthritis Res. Ther. 15, R218 (2013).

15. Santangelo, K. S., Nuovo, G. J. \& Bertone, A. L. In vivo reduction or blockade of interleukin-1beta in primary osteoarthritis influences expression of mediators implicated in pathogenesis. Osteoarthr. Cartil. 20, 1610-1618 (2012).

16. Sandoval, D. A. \& D'Alessio, D. A. Physiology of proglucagon peptides: role of glucagon and Glp-1 in health and disease. Physiol. Rev. 95, 513-548 (2015).

17. Ban, K. et al. Cardioprotective and vasodilatory actions of glucagon-like peptide 1 receptor are mediated through both glucagon-like peptide 1 receptordependent and -independent pathways. Circulation 117, 2340-2350 (2008).

18. Li, H. T. et al. Exendin-4 enhances motor function recovery via promotion of autophagy and inhibition of neuronal apoptosis after spinal cord injury in rats. Mol. Neurobiol. 53, 4073-4082 (2016).

19. Schisano, B. et al. GLP-1 analogue, liraglutide protects human umbilical vein endothelial cells against high glucose induced endoplasmic reticulum stress. Regul. Pept. 174, 46-52 (2012).

20. Zhao, X. et al. Liraglutide inhibits autophagy and apoptosis induced by high glucose through GLP-1R in renal tubular epithelial cells. Int. J. Mol. Med. $\mathbf{3 5}$ 684-692 (2015).

21. Zhang, Y. et al. Liraglutide protects cardiac microvascular endothelial cells against hypoxia/reoxygenation injury through the suppression of the SR-Ca(2 + )-XO-ROS axis via activation of the GLP-1R/PI3K/Akt/survivin pathways. Free Radic. Biol. Med. 95, 278-292 (2016).
22. Jiang, Y. Q. et al. Geniposide prevents hypoxia/reoxygenation-induced apoptosis in H9c2 cells: improvement of mitochondrial dysfunction and activation of GLP-1R and the PI3KVAKT signaling pathway. Cell. Physiol. Biochem. 39, 407-421 (2016).

23. McCulloch, K., Litherland, G. J. \& Rai, T. S. Cellular senescence in osteoarthritis pathology. Aging Cell 16, 210-218 (2017).

24. Yang, $X$. et al. Mechanical and IL-1 beta responsive miR-365 contributes to osteoarthritis development by targeting histone deacetylase 4. Int. J. Mol. Sci. 17, 436 (2016)

25. $\mathrm{Li}, \mathrm{H}$. et al. Endoplasmic reticulum stress regulates rat mandibular cartilage thinning under compressive mechanical stress. J. Biol. Chem. 288 18172-18183 (2013).

26. Busija, L. et al. Osteoarthritis. Best Pract. Res. Clin. Rheumatol. 24, 757-768 (2010).

27. Loeser, R. F., Collins, J. A. \& Diekman, B. O. Ageing and the pathogenesis of osteoarthritis. Nat. Rev. Rheumatol. 12, 412-420 (2016).

28. Aigner, T., Sachse, A., Gebhard, P. M. \& Roach, H. I. Osteoarthritis: pathobiologytargets and ways for therapeutic intervention. Adv. Drug. Deliv. Rev. 58, 128-149 (2006).

29. Heraud, F., Heraud, A. \& Harmand, M. F. Apoptosis in normal and osteoarthritic human articular cartilage. Ann. Rheum. Dis. 59, 959-965 (2000).

30. Mazzetti, I. et al. Differential roles of nitric oxide and oxygen radicals in chondrocytes affected by osteoarthritis and rheumatoid arthritis. Clin. Sci. 101, 593-599 (2001).

31. Csaki, C., Mobasheri, A. \& Shakibaei, M. Synergistic chondroprotective effects of curcumin and resveratrol in human articular chondrocytes: inhibition of IL-1 beta-induced NF-kappa B-mediated inflammation and apoptosis. Arthritis Res. Ther. 11, R165 (2009).

32. Kim, H. A. \& Blanco, F. J. Cell death and apoptosis in ostearthritic cartilage. Curr. Drug Targets 8, 333-345 (2007)

33. Echtermeyer, F. et al. Syndecan-4 regulates ADAMTS-5 activation and cartilage breakdown in osteoarthritis. Nat. Med. 15, 1072-1076 (2009).

34. Alvarez, E. et al. The expression of GLP-1 receptor mRNA and protein allows the effect of GLP-1 on glucose metabolism in the human hypothalamus and brainstem. J. Neurochem. 92, 798-806 (2005).

35. Xu, G., Stoffers, D. A., Habener, J. F. \& Bonner-Weir, S. Exendin-4 stimulates both beta-cell replication and neogenesis, resulting in increased beta-cell mass and improved glucose tolerance in diabetic rats. Diabetes $48,2270-2276$ (1999).

36. Baggio, L., Kieffer, T. J. \& Drucker, D. J. Glucagon-like peptide-1, but not glucose-dependent insulinotropic peptide, regulates fasting glycemia and nonenteral glucose clearance in mice. Endocrinology 141, 3703-3709 (2000).

37. Yao, D. D. et al. Geniposide promotes beta-cell regeneration and survival through regulating beta-catenin/TCF7L2 pathway. Cell Death Dis. 6, e1746 (2015).

38. Marzioni, M. et al. Exendin-4, a glucagon-like peptide 1 receptor agonist, protects cholangiocytes from apoptosis. Gut 58, 990-997 (2009).

39. Kamada, H., Nito, C., Endo, H. \& Chan, P. H. Bad as a converging signaling molecule between survival PI3-KJAkt and death JNK in neurons after transient focal cerebral ischemia in rats. J. Cerebr. Blood Flow Metabol. 27, 521-533 (2007).

40. Cardone, M. H. et al. Regulation of cell death protease caspase- 9 by phosphorylation. Science 282, 1318-1321 (1998).

41. Nakagawa, T. et al. Caspase-12 mediates endoplasmic-reticulum-specific apoptosis and cytotoxicity by amyloid-beta. Nature 403, 98-103 (2000).

42. Uehara, $Y$. et al. Endoplasmic reticulum stress-induced apoptosis contributes to articular cartilage degeneration via C/EBP homologous protein. Osteoarthr. Cartil. 22, 1007-1017 (2014).

43. Yusta, B. et al. GLP-1 receptor activation improves beta cell function and survival following induction of endoplasmic reticulum stress. Cell Metab. 4, 391-406 (2006).

44. Zheng, X. B. et al. SIRT1/HSF1/HSP Pathway Is Essential for ExenatideAlleviated, Lipid-Induced Hepatic Endoplasmic Reticulum Stress. Hepatology 66, 809-824 (2017).

45. Boyce, M. \& Yuan, J. Cellular response to endoplasmic reticulum stress: matter of life or death. Cell Death. Differ. 13, 363-373 (2006).

46. Zhou, Y. et al. Retinoic acid induced-autophagic flux inhibits ER-stress dependent apoptosis and prevents disruption of blood-spinal cord barrier after spinal cord injury. Int. J. Biol. Sci. 12, 87-99 (2016).

47. Wang, Q. et al. Identification of a central role for complement in osteoarthritis. Nat. Med. 17, 1674-1679 (2011). 
48. Pelletier, J. P. et al. Strontium ranelate reduces the progression of experimental dog osteoarthritis by inhibiting the expression of key proteases in cartilage and of IL-1beta in the synovium. Ann. Rheum. Dis. 72, 250-257 (2013).

49. Li, Y. et al. Free cholesterol-loaded macrophages are an abundant source of tumor necrosis factor-alpha and interleukin-6: model of NF-kappaB- and map kinase-dependent inflammation in advanced atherosclerosis. J. Biol. Chem. 280, 21763-21772 (2005)

50. Liu-Bryan, R. \& Terkeltaub, R. Emerging regulators of the inflammatory process in osteoarthritis. Nat. Rev. Rheumatol. 11, 35-44 (2015).

51. Lianxu, C., Hongti, J. \& Changlong, Y. NF-kappaBp65-specific siRNA inhibits expression of genes of COX-2, NOS-2 and MMP-9 in rat IL-1beta-induced and TNF-alpha-induced chondrocytes. Osteoarthr. Cartil. 14, 367-376 (2006).

52. Gou, S. et al. Glucagon like peptide-1 attenuates bleomycin-induced pulmonary fibrosis, involving the inactivation of NF-kappaB in mice. Int. Immunopharmacol. 22, 498-504 (2014).

53. Guo, C. et al. Glucagon-like peptide 1 improves insulin resistance in vitro through anti-inflammation of macrophages. Braz. J. Med. Biol. Res. 49, e5826 (2016).

54. Shiraki, A. et al. The glucagon-like peptide 1 analog liraglutide reduces TNFalpha-induced oxidative stress and inflammation in endothelial cells. Atherosclerosis 221, 375-382 (2012).

55. Dai, Y., Dai, D., Wang, X., Ding, Z. \& Mehta, J. L. DPP-4 inhibitors repress NLRP3 inflammasome and interleukin-1 beta via GLP-1 receptor in macrophages through protein kinase C pathway. Cardiovasc. Drugs Ther. 28, 425-432 (2014).
56. $L i, H$. et al. Mechano growth factor-E regulates apoptosis and inflammatory responses in fibroblast-like synoviocytes of knee osteoarthritis. Int. Orthop. 39 2503-2509 (2015).

57. Hosseinzadeh, A. et al. Apoptosis signaling pathways in osteoarthritis and possible protective role of melatonin. J. Pineal Res. 61, 411-425 (2016).

58. Zhang, K. \& Kaufman, R. J. From endoplasmic-reticulum stress to the inflammatory response. Nature 454, 455-462 (2008).

59. Hu, P., Han, Z., Couvillon, A. D., Kaufman, R. J. \& Exton, J. H. Autocrine tumor necrosis factor alpha links endoplasmic reticulum stress to the membrane death receptor pathway through IRE1alpha-mediated NF-kappaB activation and down-regulation of TRAF2 expression. Mol. Cell. Biol. 26 3071-3084 (2006).

60. Xue, E. X. et al. Pterostilbene inhibits inflammation and ROS production in chondrocytes by activating Nrf2 pathway. Oncotarget 8, 41988-42000 (2017).

61. Jiang, L. B. et al. Adipose-derived stem cells induce autophagic activation and inhibit catabolic response to pro-inflammatory cytokines in rat chondrocytes. Osteoarthr. Cartil. 24, 1071-1081 (2016).

62. Pfaffl, M. W. A new mathematical model for relative quantification in real-time RT-PCR. Nucleic Acids Res. 29, e45 (2001)

63. Chu, X. et al. Protective effect of lentivirus-mediated siRNA targeting ADAMTS 5 on cartilage degradation in a rat model of osteoarthritis. Int. J. Mol. Med. 31, 1222-1228 (2013)

64. Moskowitz, R. W. Osteoarthritis cartilage histopathology: grading and staging Osteoarthr. Cartil. 14, 1-2 (2006). 